\title{
E-Maturity and School Development: When the Tail Wants to Wag the Dog
}

\author{
Peter Micheuz \\ University Klagenfurt, Institute for Informatics Systems, 9020 Klagenfurt, Austria \\ peter.micheuz@uni-klu.ac.at
}

\begin{abstract}
E-maturity as synonym for successful integration of digital technologies in schools can be seen as the golden thread of this paper. It deals with an Austrian case study about recently conducted ICT-certifications within a ministerial e-Learning project. The process of awarding successful Austrian schools with ICT-certificates is founded on an evaluation framework which has been developed independently from other similar European approaches. Finally, it will be argued that understanding the interdependency of successful ICT integration and school development is a key issue to achieve the status of ematurity.
\end{abstract}

Keywords: ICT in education, Certification, e-Learning, e-Maturity.

\section{Introduction}

Very often ICT implementation at school level is still driven by enthusiastic teachers, and the majority of schools can be stated to be on a low maturity level, where ICT is not strategic but something that only innovators take care of. This often results in uncoordinated use of ICT at school where digital technology is not seen as a part of a general strategy. A large-scale study for the Scandinavian countries [1] shows that many schools have developed an ICT strategy, but it was assessed that in many cases the strategies were developed years ago, and that they are not linked with other strategies, nor are they widely known among the stakeholders. Instead, digital technologies need to be integrated into a comprehensive concept of school development which supports the main goals and functions as a catalyst for change.

Probably it is the lack of sustainable guiding strategies for the roll out of the technology that ICT does not raise learning standards and learner's achievement [2]. A more fundamental reason may lie in the inherent malfunction of digital media within the present educational structure which is still based on the industrial paradigm [3].

Before addressing the Austrian case study of successful intervention of implementing digital technologies and e-learning in schools, I want to point out two different perspectives on innovation diffusion as a framework for further analysis.

Two different approaches - digital technologies as a catalyst for reform and that of a lever for reform - can be distinguished.

The catalyst pattern provokes or instigates far-reaching and sustainable change which exceeds initial intentions. No miracles derive from the mere presence of ICT in 
a school; it does not, except in unusual circumstances, act as a catalyst for wide scale improvements. Just "throwing technology into the classroom and hoping for good things" is not a good strategy to get a better teacher in the digital age [4].

However, ICT can be a powerful lever for change. In contrast to the catalyst pattern, the lever pattern implies that ICT is not used as an agent but as a tool. An agent, once introduced, can act beyond its immediate goal. A lever can only be applied to achieve an intended goal [5].

These different models are critical for policy which applies for large-scale national investments in IT infrastructure and in-service trainings as well as for the autonomously school administrations when they plan to invest in digital technologies without objectives for pedagogical improvements.

\section{Structuring and Benchmarking ICT Integration in Schools}

"Schools have now got unprecedented access to ICT tools to support teaching and learning. However, research indicates that many educational institutions fail to make full use of their ICT facilities, while at the same time ICT still has the potential for a positive impact on the education system" [6]. Despite many - technologically - fairly well-developed schools in Austria, there is evidence that these schools can be in fact considered as e-ready but not as e-mature [7]. Becta, UK, defines institutional ematurity (sometimes described as 'e-enablement') as the capacity and capability of a college or learning institution to make strategic and effective use of technology to improve educational outcomes [8].

Even with the best (national) education system possible, much activity in schools is determined autonomously by the school itself, its management and its pedagogical staff and by the students. Embedded in a competitive culture in the educational area of some countries, there already exist (independent) approaches to assess whether schools are e-mature.

Based on a self-review framework, schools from the UK can apply for the so called "ICT Mark", a national accreditation scheme which recognizes them for their achievements in reaching a standard of maturity in their use of technology.

The (online) self-review framework is the backbone for schools to benchmark themselves in terms of ICT integration, to draw up action-plans and to review their progress over time.

In order to be awarded the ICT Mark schools need to show that they meet the threshold standards in:

1. Leadership and Management - ICT vision and strategy

2. ICT in the curriculum

3. Learning and Teaching with ICT

4. Assessment of and with ICT

5. Professional Development

6. Extending opportunities for learning

7. Resources - provision, access and management

8. Impact on pupil outcomes

Moreover, beyond recognizing well performing schools in form of this certification, Becta rewards the best schools with the ICT Excellence Award in the 
categories: best whole school; beyond the classroom; leadership; management and collaboration; learning experience and support for schools.

Another self-assessment tool, the European Framework for the Evaluation of ICT in Education, was developed as part of the P2P-Inspectorates from six countries [9]. Assessing ICT integration in schools from an holistic perspective, this project was undertaken by the P2P-Inspectorates and is available at the website of the European School Net [10].

This framework consists of three main themes: Conditions, Use and Outcomes. Conditions

C1. Leadership

C2. Infrastructure and access

C3. Curriculum planning

C4. Quality assurance and improvement

Use

U1. Pupil use

U2. Teaching process

U3. Administrative use

Outcomes

O1. Impact on learning and standards

Twenty quality indicators with nearly 100 corresponding evidence pointers are identified within each quality area. This framework can be used in form of a checklist with the marks 1 (poor), 2 (insufficient), 3 (sufficient) and 4 (good) resulting from interviews with school management, teaching staff and students, by observations of lessons and by document review and student products.

A third self-evaluation instrument has been developed in Belgium [11]. This assessment framework draws on the EFQM excellence model which helps organizations, like schools, to determine at which point they find themselves on their way to excellence. Schreurs distinguishes three stages in the evolution of ICT at schools.

The first stands for basic principles in using the computers by teachers, the second for applying the computer as a passive and active medium such as the Internet and using it in various contexts. The third phase encompasses the seamless integration of ICT across the curriculum and learning activities where the focus lies on the learner.

The structure of the framework is divided into 5 sections:

1. The vision for ICT use in school (a strategy to achieve the ICT vision)

2. Secondary processes (school organization and management, ICT coordinators)

3. Resources (ICT infrastructure, government regulations, funding programs)

4. Primary processes (curriculum development, Integration of ICT)

5. Desired results (results for the learner, teacher, parents, society and government) 


\section{The Austrian Project eLSA}

One major national Austrian top-down initiative, called eLSA, which is an acronym for "e-Learning in everyday school live" has been launched by the Ministry of Education in 2002. Apparently, within six years eLSA has become a trademark for successful integration of e-Learning for the lower secondary level (pupils are 10-14 years old). Even if there is less financial stimulation as it was at the beginning, the number of schools is still increasing and profiting from the enormous practical knowledge which has been gathered by innovative teachers within several years of classroom experience. By now about 60 out of 330 academic secondary schools take part. Each school involved in the eLSA-project has been financially supported for hardware and in-service training. Simultaneously they had to commit themselves to deliver regularly strategy plans and annual reports, and above all, to aim at the ambitious eight project goals.

1. Each student has to get in touch with e-Learning and has to try out "e-Learning sequences" in lower secondary education.

2. Each teacher has to experience e-Learning sequences in his/her own subjects (in at least one subject) and has to share his/her experience with all members of the teaching teams involved in these subjects and the participating classes.

3. Within their subject area, teachers have to discuss the potential and limits of eLearning.

4. eLSA schools develop concrete models for evaluating e-Learning. They cooperate and share their experiences with other schools.

5. The school program should explicitly contain (revised) e-Learning aspects.

6. e-Learning must be an important concern of the school administration. The project has high priority in the school routine.

7. A steering group coordinates and harmonizes the "e-Learning" content developments, ensures their practical application and the progress of the project.

8. The school offers its students the possibility to obtain at least one IT or eLearning certificate (on a voluntary basis).

It is important to enumerate these goals explicitly. They have little to do with clear and easy instructions for implementing ICT. Moreover, the goals 3, 5 and 7 aim directly at the existence of efficient structures of school development. Goal 3 assumes functioning collaboration among teachers, goal 5 needs an elaborated and an executed school program. Goal 7 demands knowledge and experiences in school governance in terms of controlling big projects at schools [12].

\section{4 eLSA and School Development}

The inherent nature of organizations can be considered conservative. They are protecting themselves from constant change [13]. At an early stage, Schön recognized the need for a "learning organization". As part of its improvement process, it is necessary that they make a transition from an "I-culture" to a "We-culture." This 
draws on Fullan's view [14] that "Learning on the job is the sine qua non of improvement."

By examining the theory of diffusion of innovation [15], change in schools can be better understood. Now and in the future, schools are faced with an unprecedented rate of change, forced by the "grown up digital" [4].

There is evidence that especially among teachers we can disproportionally find many who are resistant to personal and professional development. Moreover, it seems that the population of teachers in many schools perfectly confirms Roger's adoption/innovation curve with very few innovators, some early adopters, with a big late majority and a few laggards.

The ironical saying "Schools change slower than churches" from the American school researcher Richard Gross, Stanford, can be considered as the pedagogical analogy of the physical law of inertia.

ICT implementation at a school level should be viewed in the context of school improvement plans and not simply as a technical issue. The highest returns on ICT in education appear to come when ICT is seen as part of a holistic approach within a clearly defined school development strategy. Success of digital technologies in schools depends on appropriate embedding in didactical settings. This cannot be seen independently from an overall discussion of school quality. This holistic view must be taken into account when thinking of using new media for educational purposes.

Digital technologies can be convenient media for continual professional development through collaboration, capture and diffusion of tacit knowledge, and data recording, analysis, and display. Truly, many schools investigated in the OECD study [5] have implemented an IT infrastructure that support these ends; however, only a few report professional development outcomes from such applications.

There will always be a risk in adopting ICT solutions when the tail (ICT) wags the dog (the organization school). ICT is there to serve the needs of education, not the other way round. Successful implementation of ICT greater depends on the personalities of headmasters and teachers, They are the key players of "change" and responsible for (autonomous) school improvement.

Especially this aspect was strongly underestimated when the eLSA project as a driver for innovation processes was launched. In other words, some schools were not prepared well enough [12].

\section{Recognizing e-Mature eLSA-Schools}

As part of the "FutureLearning" program of the Austrian Ministry for Education [16] eLSA is still an active network driven by a motivated team, although fighting with decreasing budgets. After six years it was time to recognize and distinguish those schools which not only aimed at the goals set by eLSA, but also fairly tried to achieve them. This was the main rationale for developing the eLSA-certificate.

Having visions and defining ambitious goals to achieve is just one side of the coin. Finding descriptors and indicators for a valid and viable certification process, where theory meets practice is the other. Consequently, almost simultaneously and independently from the three already existing approaches in the UK, Belgium, and the P2P project within the European Schoolnet and described above, an Austrian 
evaluation framework for assessing and recognizing outstanding eLSA schools, has been developed.

By now, the eLSA-certificate is voluntary and the pilot eLSA schools applied for it. The evaluation and certification processes for these schools were conducted by national and regional eLSA-coordinators and then successfully completed in 2008.

So far, in Austria, except for very few vocational schools which are ISO 9001 certified, there is no experience with a certification process of this kind. Moreover, external evaluations have no noteworthy tradition in Austria's educational system, and inspectors are seen rather as managers and advisers than as external auditors.

Each certification and its preceding evaluation process require objectives and indicators. Based on the eight eLSA goals, the current evaluation framework encompasses more than 60 detailed descriptors with corresponding indicators [17].

This table impressively illustrates the hurdles and pitfalls of putting a (theoretical) framework into action and practice, especially when we look closer at the descriptors and indicators and bear in mind how to monitor and validate them. Executing the certification process reveals problems which arise by interpreting and quantifying indicators accurately. In some cases headmasters have been deterred by this framework. After silencing their scruples of failing to meet all requirements for being awarded with the eLSA-certificate, by the end of the year about twenty applied for it.

Questions which arise in the context of this framework may be: Are the terms "eLearning" and "learning sequences" defined sharply enough? Both terms leave a wide room for interpretation. Does the school record all protocols and formal in-service trainings of the teachers? Do the class registers show adequate information?

Table 1. Exemplary descriptors and indicators for the second eLSA goal: Each teacher has to experience e-learning sequences in his/her own subjects (in at least one subject) and has to share his/her experience with all members of the teaching teams involved in these subjects and the participating classes

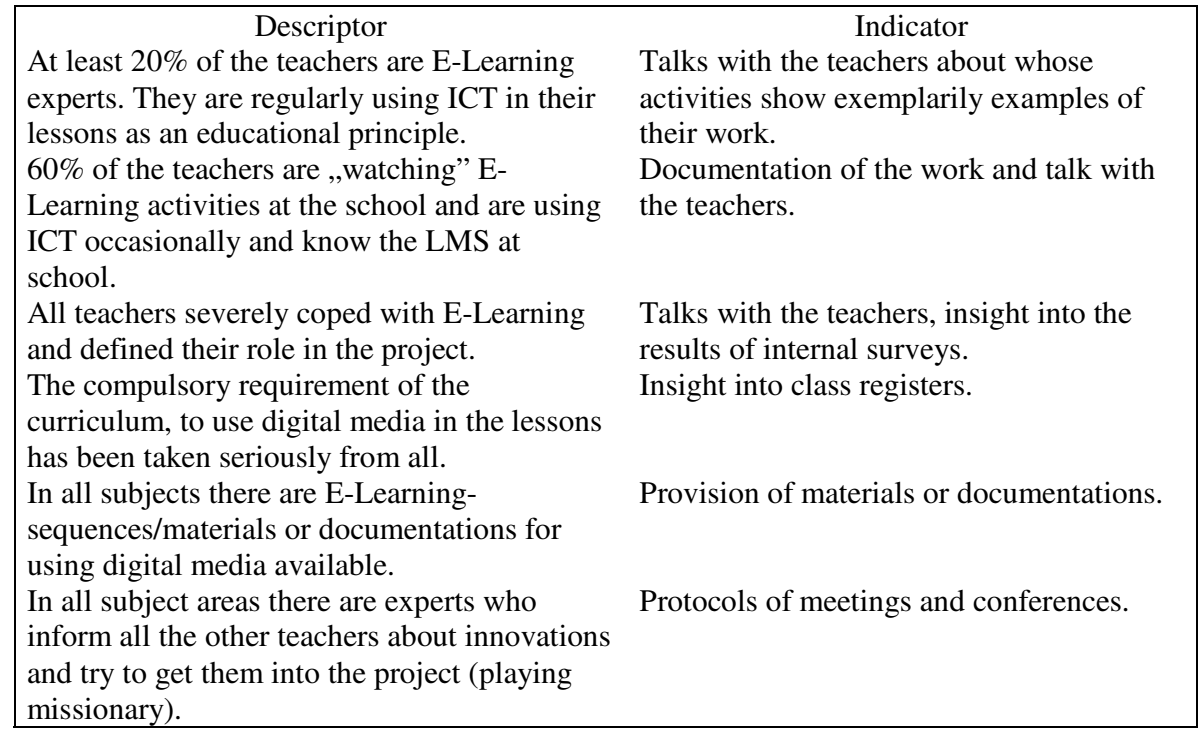


The first questions represent a qualitative problem, whereas the other addresses the quantitative issue if school administration has all relevant information about teachers at hand.

Already the very first proposed detailed objective "All pupils have verifiably come into contact with e-learning" brings up the question of interpreting the term e-learning and, moreover, the term "verifiably". Considering an average school with several hundred pupils at lower secondary education level, validating this indicator is hardly manageable in time and within reasonable costs.

Another detailed objective, for example, affects the teachers' e-literacy. The proposed indicators are teachers' certificates, as the ECDL (European Computer Driving License) and attendance at in-service trainings. Normally, not even these data about hard facts are easily available at schools.

Other goals as "There is a pronounced culture of communication in the area of elearning" and the corresponding indicators "conference protocols and insight into online-communications" represent rather a qualitative than a quantitative challenge and require records of all formal and informal meetings.

Probably this theoretical framework of descriptors will soon be revised by a feedback loop. First experiences showed that some indicators turned out to cause an unwarrantable expenditure in time and costs for the inspectors and an administrative overload for the schools.

\section{Certification Procedure and Empirical Findings}

The theory of structuring, evaluating and benchmarking (successful) ICT integration in schools is important and has independently resulted in different, although not too divergent European frameworks. They all have to prove themselves by practical feasibility.

Whereas already about one thousand of schools throughout the UK have been rewarded by Becta's ICT Mark, the eLSA certification in Austria is still in an initial phase.

Recently, in the last month of 2008, the author took part in three certification processes with the following procedural structure:

1. The particular school determines if the goals are reached and applies for the eLSA-certificate at the regional eLSA-coordinator.

2. The regional coordinator conducts a preliminary visit and talks with school administration and fixes a day for the certification.

3. The chronology of the day of certification, where the regional eLSA coordinator in charge brings in an external expert is as follows:

- Talk with the headmaster, the system administrator and the eLSAcoordinators of the school.

- Meeting with a representative sample of pupils and students who show eLearning in context and report about experiences in a computer lab.

- Meeting with a representative sample of teachers involved.

- Concluding meeting with the eLSA key players at the particular school, and the announcement of the results of the inspection.

4. Finally, the school gets a report from the regional eLSA-coordinator. 
So far, the school audits yielded most valuable, but not necessarily surprising empirical results.

- eLSA-certified schools have already fairly good preconditions in terms of the IT infrastructure and engaged ICT teachers before the eLSA project.

- eLSA-certified schools are led by extraordinary supportive headmasters with (clear) visions and a sense of accountability.

- The IT-infrastructure in eLSA schools is maintained by very engaged system administrators who complain about the lack of a sufficient IT infrastructure and low budgets.

- Cooperation among teachers is still underdeveloped.

- Many teachers use computers in their lessons, but often only to show and distribute digital material.

- The dominating learning platform, with a fairly well usage is Moodle. However, most courses lack interactive and collaborative activities.

- Many special ICT related activities in various subjects could be observed.

- Almost all pupils explicitly expressed their joy with e-Learning. They liked working with computer because "one learns differently", "it is a nice variety in view of everyday school", "it is practical because we have less paper", "you get quick feedback", "you can choose exercises individually".

These statements encompass the answer to the frequently asked question about the additional value of ICT integration. Here it is: It is joy, variety, individuality and a learner centered approach. Sometimes effective answers can be simple.

Further impacts of e-Learning with respect to "an improved and efficient learning" could not be recognized. Discussing Becta's definition of e-maturity, which aims at the improvement of educational outcomes, could be a starting point for another paper. The author is quite aware of the fact that not one single school achieved all - perhaps too idealistic - goals of the eLSA-certificate.

Viewed from a pragmatic perspective, the eLSA coordinators consider the awarding of e-mature schools in this initial phase as an important undertaking. It is a necessary appreciation for innovative schools to make visible school development which has been put into practice.

\section{Conclusions}

It seems to be common sense that a rapidly changing world needs schools as learning organizations with an adequate use of digital technologies. Actually, the whole arsenal of digital technologies leads us to believe in their inherent potential of improved learning. But the fact is that successful school development (in terms of ICT) does not depend on perfect technology but on imperfect humans, teachers and students included.

As pointed out, digital technologies can exploit their full potential in education, however, only if the underlying mindsets aim at school development. It is simply more effective, when the dog wags the tail.

The interdependence of technology and education tends to be a pedagogical challenge with moving targets, according to the message "What is not measured will 
not change" [18]. There is hope that the spread of the Austrian eLSA-certificate, which benchmarks the e-maturity of schools against each other, can raise the educational efficacy of digital technologies.

\section{References}

1. Pedersen, S., et al.: E-learning Nordic 2006, Impact of ICT on Education, Ramboll Management, Copenhagen (2006)

2. Hughes, W.D.: An Overview of the Potential, Constraints an Experience of Developing a Coherent Strategy for the Use of Informatics to Raise Learning Standards in Secondary Schools Based on UK Custom and Practice. In: Micheuz, et al. (eds.) Inonovative Concepts for Teaching Informatics, Ueberreuter, Vienna, pp. 136-143 (2005)

3. Albirini, A.: The Crisis of Educational Technology, and the Prospect of Reinventing Education. Educational Technology \& Society 10(1), 227-236 (2007)

4. Tapscott, D.: Grown Up Digital. McGraw-Hill, New York (2008)

5. Venezky, R.: Quo Vademus? The Transformation of Schooling in a Networked World (2002)

6. Durando, M., et al.: eMature Schools in Europe, Report European Schoolnet (2007)

7. Micheuz, P.: E-Readiness in Austrian Schools - From Theory and Visions to Practice and Reality. In: Proceedings of LYICT, Kuala Lumpur (2008)

8. Becta (2008), http: / / learningandskills.becta. org .uk/display. $\mathrm{cfm}$ ?page=1897

9. Hogenbirk, P.: ICT Action School Development on the Basis of an Inspectorate Assessment. In: Proceedings of LYICT, Kuala Lumpur (2008)

10. EUN: European Schoolnet, http: / / www . eun. org

11. Schreurs, J.: ICT Use in School: Vison and Performance Measures. In: Proceedings of the ICL-conference, Villach (2007)

12. Weiser, B.: Zusammenfassende Analyse des eLSA Projekts aus Schulentwicklungsperspektive (2005),

http://elsa.schule.at/evaluation-ergebnisse/weiser.pdf

13. Schön, D.: Beyond the Stable State. Public and private learning in a changing society. Penguin (1974)

14. Fullan, M.: The NEW Meaning of Educational Change, 3rd edn., p. 239. Teachers College Press, New York (2001)

15. Rogers, E.M.: Diffusion of Innovations, 4th edn. The Free Press, New York (1995)

16. BMUKK, FutureLearning - the successful next development of the eFit initiative (2008), http: / /www.bmukk.gv.at/medienpool/17141/konzept_en_kurz.pdf

17. ELSA, Zertifizierungshilfe, http://elsa20.schule.at/uploads/media/ elsa_zertifizierung_orientierungshilfe_01.pdf

18. Rieke-Baulecke, T., et al.: Schulmanagement. Westermann, Braunschweig (2006) 